\title{
Le Officine del Minotauro
}

Diventare adulti oggi è un processo intrapsichico che porta il singolo a riflettere sulle proprie caratteristiche personali così come sulla complessa e precaria realtà sociale in cui viviamo, sviluppando competenze e abilità specifiche. La diffusione della tecnologia, inoltre, sta portando a una profonda e radicale rivoluzione culturale e cognitiva tanto da creare una difficile sincronia tra contesti istituzionali di apprendimento e mondo del lavoro. Infine, i dati statistici dichiarano un aumento di giovani in ritiro scolastico e sociale accompagnati da sentimenti di inutilità, vergogna e solitudine.

Alla luce del panorama socioeconomico contemporaneo, che come ambiente di crescita nella Comunità incide direttamente sulle coordinate di funzionamento psicologico degli individui, l'équipe Apprendimento del Minotauro ha avviato il progetto Officine, riunendo una serie di dispositivi di cura sperimentati negli ultimi venti anni e finalizzati a supportare la costruzione identitaria dei giovani come individui e come persone inserite nel proprio ambiente di vita. Officine, in questa prospettiva, ha l'obiettivo di accompagnare e sostenere il giovane nella ripresa e nella creazione di un futuro pensabile, sia personale che lavorativo.

Nello scenario attuale, i giovani che faticano a crescere e occupare un ruolo nel complesso mondo odierno vengono chiamati NEET: 'giovani che rifiutano qualsiasi forma di educazione, di inserimento sociale e di lavoro'. All'interno di questo gruppo troviamo adolescenti e giovani adulti in una situazione di blocco più o meno severo, disorientati fra le possibili scelte, preoccupati e demotivati dal possibile fallimento, sofferenti per la situazione di stallo. Questa dinamica si manifesta attraverso la rinuncia ad apprendere, nella problematicità della scelta scolastica o universitaria e, in generale nel ritiro degli investimenti: il bisogno di fermarsi per costruire percorsi

\footnotetext{
*Psicologa psicoterapeuta; Vicepresidente della Cooperativa Minotauro e referente della sede Minotauro di Padova; Ideatrice del progetto Officine, responsabile dell'équipe Apprendimento e Laboratori, direttrice del master 'Adolescenti in crisi'.

E-mail:kprovantini@gmail.com ; sito: www.katiaprovantini.com
} 
significativi è percepito come una necessità imprescindibile, rischiando tuttavia di bloccarsi nella percezione di non aver nulla da sviluppare, nessuna qualità su cui appoggiarsi e alcun valore da difendere. Secondo quanto segnalano Istat e Unicef, i Neet in Italia hanno superato i 2 milioni (dati del 2018 in continuo aumento) e, tra i 15 e i 29 anni, i giovani che non studiano e non lavorano sono stati il 23,4\%: fenomeno allarmante, molto presente sia tra i maschi che tra le femmine, oltre ad essere in aumento per entrambi. Secondo quanto osservato dall'Istituto Minotauro, questi ragazzi faticano a progettare il proprio futuro per un'impossibilità di pensarlo in termini positivi. Il futuro, che si rappresenta oggi precario e minaccioso, ha la capacità di interferire a più livelli nella vita degli individui, dai piani di sviluppo individuale fino ai miti culturali che influenzano le logiche educative, la relazione tra generazioni e l'intero impianto su cui fondare Comunità educante e Comunità sociale.

Dal punto di vista clinico è sempre più evidente come un futuro difficoltoso vada ad intaccare la capacità di investire in progetti del presente. I ragazzi hanno sviluppato un linguaggio che racconta il 'non senso' di certi impegni, certe logiche e certe richieste. Per quel che riguarda il rapporto tra generazioni, il futuro interpretato positivamente o negativamente è in grado di modificare la relazione tra generazioni: le richieste e le indicazioni che gli adulti rivolgono ai giovani variano a seconda che il futuro si percepisca lineare e raggiungibile facilmente, oppure, minaccioso e incerto.

Parallelamente, in un'epoca complessa l'identità adulta si ricerca quando si è in grado di porsi domande giuste, di senso, sulla propria esistenza, ma anche quando si sviluppano capacità progettuali, decisionali, narrative, riflessive, per cercare di aprire prospettive di senso possibili.

La tematica, vista da questo punto di vista, ci aiuta a mettere a fuoco che i ragazzi, nel rapporto con gli adulti, hanno bisogno di dialoghi di senso e di valore. Non dobbiamo dimenticarci che gli adolescenti di oggi sono figli di un modello educativo profondamente centrato e alimentato dalla relazione, nella quale la famiglia affettiva ha alimentato un continuo dialogo intergenerazionale. Allo stesso tempo purtroppo la famiglia relazionale, funzionale per i compiti evolutivi dell'infanzia, trova la sua maggiore difficoltà nel supportare lo sviluppo adolescenziale centrato sui compiti di separazione, individuazione e differenziazione. La difficoltà aumenta se, come dicevamo, il futuro è minaccioso e la Società è percepita come problematica.

Le Officine del Minotauro si collocano in uno spazio al contempo psichico e sociale; sono un progetto a sostegno della crescita dei giovani, propone loro nuove sperimentazioni per la costruzione dell'identità adulta $\mathrm{e}$ fornisce agli adulti di riferimento gli strumenti necessari per aiutare i ragazzi nella costruzione del proprio futuro. Officine è inoltre un movimento di condivisione per costruire e diffondere un nuovo paradigma culturale. Il progetto è strutturato intorno al fare: un fare dotato di senso, in grado di 
riavviare la speranza e supportare la ripresa dei processi di mentalizzazione. Lontano tanto dalle teorie occupazionali quanto dalla semplice espressività solitaria e narcisistica, il fare diviene opportunità di costruzione identitaria: esperienza trasformativa in grado di riabilitare l'immagine di sé mortificata e di sperimentare processi creativi condivisi e radicati nell'incontro con l'altro e nella realtà sociale.

Il progetto Officine, con supervisione scientifica di Katia Provantini e coordinamento di Alessia Lanzi, si rivolge principalmente a ragazze e ragazzi (16-27 anni) che, superato il periodo di obbligo formativo scolastico, vivono una fase di stallo: fatica nella frequenza scolastica, disorientamento nella scelta della scuola o dell'università, blocco in preparazione della maturità o di sessioni universitarie, difficoltà nella ricerca di lavoro; oppure situazioni stazionarie senza scuola, né lavoro, né altre formazioni in corso. Per permettere ai ragazzi di ri-accedere al proprio progetto di vita e rendere pensabili nuovi percorsi oltre la crisi, Officine propone a ciascuno percorsi personalizzati costruiti grazie all'ausilio di un tutor individuale.

Il dispositivo maggiormente proposto è il Laboratorio (Mittino \& Provantini, 2017): strumento di presa in carico finalizzato al superamento dei blocchi del pensiero e al riavvio dei processi di crescita sul piano emotivo, affettivo, relazionale e cognitivo; si struttura attorno al fare, che diventa mezzo per accedere a nuove rappresentazioni di sé, riattivare i processi di pensiero bloccati e sperimentare esperienze emotivamente valorizzanti.

I laboratori possono essere individuali o di gruppo, e uniscono aspetti espressivo-creativi, di apprendimento e di supporto alla crescita. Accanto agli incontri individuali, sono previsti i laboratori gruppali (di cinema, identità e ruolo professionale, patente di guida, competenze trasversali o soft skills, scelta universitaria, costruzione curriculum, preparazione agli esami, etc.) e i Laboratori del cambiamento (mentoring) che prevedono la collaborazione tra generazioni di professionisti e l'integrazione di approcci nel superamento delle difficoltà.

Al fine di proporre interventi che non siano limitati alle difficoltà di crescita dei singoli ragazzi, Officine si rivolge alla Comunità Educante proponendo un nuovo paradigma di supporto alla crescita che coinvolge numerosi interlocutori. Azioni specifiche (formazione, sensibilizzazione, laboratori dedicati, progettazione di tirocini) coinvolgono genitori, operatori psicosocio-sanitari, docenti e referenti delle aziende.

La necessità è di sviluppare un movimento condiviso di trasformazione delle logiche lineari in logiche complesse e interconnesse. Tutto ruota intorno ai nuovi paradigmi generati dai cambiamenti repentini del mondo sociale e dalle trasformazioni tecnologiche: dalla conoscenza, che da contenuto diviene processo, alla funzione dell'adulto che, da saggio e detentore di risposte, si trasforma in colui che sostiene la speranza e il valore intrinseco della domanda e del cambiamento. Officine da questo punto di vista vuole 
dare testimonianza del ruolo fondamentale assunto dal fare condiviso: in un epoca di forte destabilizzazioni, in cui un certo modo di stare al mondo sembra chiudersi e i nuovi riferimenti non risultano ancora radicati, gli adulti devono poter sostare nel cambiamento e acquisire tutte quelle competenze necessarie al buon esito del viaggio. Un viaggio che per molti aspetti nessuno di noi ha precedentemente sperimentato e di cui non esistono mappe o diari di viaggiatori rientrati in porto. Potremmo dichiarare l'impossibilità di partire, non avendo mete né strumenti. Oppure possiamo immaginare di sfruttare le esperienze e le competenze acquisite per far emergere un nuovo modo di essere adulto, maggiormente generativo, più consapevole dei propri funzionamenti, più interessato e responsabile delle differenze che non delle somiglianze, meno soggiogato dalla paura e dalle abitudini. Dal punto di vista di Officine, tutto ciò consentirebbe di creare, insieme, quei ponti necessari tra l'immobilità della crisi attuale e il futuro che i giovani e la Società sono chiamati ad immaginare prima ancora di costruire.

Tutte le informazioni e i contatti sono disponibili al sito: https://officine.minotauro.it/

\section{BIBLIOGRAFIA}

Mittino, F., \& Provantini, K. (a cura di) (2017). Apprendere: un processo complesso. Psichiatria e Psicoterapia, XXXVI(1), marzo 2017. Disponibile: https://www.fioritieditore.com/prodotto/psichiatria-e-psicoterapia-no-1-marzo-2017/

Conflitto di interessi: l'autore dichiara che non vi sono potenziali conflitti di interessi.

Approvazione etica e consenso a partecipare: non necessario.

Ricevuto per la pubblicazione: 20 marzo 2021.

Accettato per la pubblicazione: 22 marzo 2021.

${ }^{\circ}$ Copyright: the Author(s), 2021

Licensee PAGEPress, Italy

Ricerca Psicoanalitica 2021; XXXII:537

doi:10.4081/rp.2021.537

This article is distributed under the terms of the Creative Commons Attribution

Noncommercial License (by-nc 4.0) which permits any noncommercial use, distribution,

and reproduction in any medium, provided the original author(s) and source are credited. 